\title{
A convenient telescope performance metric for imaging through turbulence
}

\author{
George Z. Angeli*a ${ }^{\mathrm{a}}$, Byoung-Joon Seo ${ }^{\mathrm{b}}$, Carl Nissly ${ }^{\mathrm{b}}$, Mitchell Troy ${ }^{\mathrm{b}}$ \\ ${ }^{a}$ Thirty Meter Telescope Observatory, Pasadena, CA, USA 91125 \\ ${ }^{\mathrm{b}}$ Jet Propulsion Laboratory, Pasadena, CA, USA 91109
}

\begin{abstract}
This paper provides an overview of the various image quality metrics used in astronomical imaging and explains in details a new metric, the Normalized Point Source Sensitivity. It is based on the Equivalent Noise Area concept, an extension of the EE $80 \%$ metric and is intuitively linked to the required science integration time. As it was proved in recent studies, the PSSN metric properly accounts for image degradation due to the spatial frequency content of a given telescope aberration and the effects of various errors can be multiplicatively combined, like those expressed in Central Intensity Ratio. Extensions of the metric for off-axis imaging and throughput degradation are presented. Wavelength and spatial frequency dependence of PSSN are discussed. While the proper calculation of the PSSN metric requires the precise knowledge of the PSF of both the optics and atmosphere, there is a straightforward approximation linking PSSN to the Zernike decomposition of the OPD. Besides the summary of various aspects of the Point Source Sensitivity, the paper provides many numerical examples derived for the Thirty Meter Telescope.
\end{abstract}

Keywords: Systems engineering, image quality, performance estimate

\section{INTRODUCTION}

The Thirty Meter Telescope Observatory is an international collaboration for developing, constructing, and operating a ground based Extremely Large Telescope with highly segmented primary mirror. The size and budget of the project, as well as its geographically and organizationally distributed nature requires solid system engineering principles and practice. In particular, advanced performance allocation and integrated performance estimates are essential for sound requirements engineering.

The basis of relevant and reliable performance allocation and estimates is a metric that

i. Properly reflects the science capability and efficiency of the observatory, while

ii. Facilitates accurate combination of various performance components, i.e. a correct performance/error budget.

While numerous aspects of the observatory performance need to be tracked and balanced between subsystems, undisputedly the most important of those is image quality. There are two types of image quality metrics: one based on intensity loss at the center of the image, the other accounts for the area of the image.

The Strehl Ratio $(S)$ is the classic metric for central intensity loss of an image with Point Spread Function $(P S F)$ of $h$ and corresponding Optical Transfer Function $(O T F)$ of $\mathcal{H}$. (The $P S F$ is a function of the two dimensional angle $\Omega$, while the $O T F$ is a function of the two dimensional angular frequency of $v$.)

$$
S=\frac{h_{\text {actual }}(0)}{h_{\text {perfect }}(0)} \equiv \frac{\int_{\infty} \mathcal{H}_{\text {actual }} d v}{\int_{\infty} \mathcal{H}_{\text {perfect }} d v}
$$

*gangeli@tmt.org; phone 1520 318-8413; fax 1520 318-8590; tmt.org

Optical Modeling and Performance Predictions V, edited by Mark A. Kahan, Proc. of SPIE Vol. 8127, $812709 \cdot$ ㅇ 2011 SPIE · CCC code: 0277-786X/11/\$18 · doi: 10.1117/12.896919 
Here the subscript perfect refers to the diffraction limited, i.e. the perfect image of the telescope. The Strehl Ratio is a practical metric for small aberrations with short correlation length in the wavefront, as it is the case for space telescopes or adaptive optics corrected ground based telescopes. For those kinds of errors, the Strehl Ratio is connected to the wavefront variance $\left(\sigma_{\phi}^{2}\right)$ through the Maréchal approximation.

$$
S \approx e^{-\sigma_{\phi}^{2}} \approx 1-\sigma_{\phi}^{2}
$$

However, for observations with large ground based telescopes without adaptive optics, where image degradation is dominated by atmospheric seeing, the Strehl Ratio is very small; its measurements and calculations are error prone. To resolve this problem, Dierickx [1] proposed a variant of the Strehl Ratio, called the Central Intensity Ratio (CIR), which is normalized to the PSF of the perfect telescope looking through the atmosphere.

$$
C I R=\frac{h_{\text {actual }}(0)}{h_{\text {atmosphere }}(0)} \equiv \frac{\int_{\infty} \mathcal{H}_{\text {actual }} d v}{\int_{\infty} \mathcal{H}_{\text {atmosphere }} d v} \leq 1
$$

The actual PSF includes all the telescope aberrations and the atmosphere, while atmosphere denotes atmospheric seeing with a perfect telescope. Castro et al. [2] reported that the Central Intensity Ratio is multiplicative with good approximation for various error sources, i.e. it can be used for multiplicative error budgeting.

$$
C I R_{\text {total }}=\prod_{i} C I R_{i}
$$

However, for large aberrations, the actual shape of the PSF is also important, not just the energy loss of the image core. Image size type metrics are capturing this additional information better. As it will be shown later, these metrics have more straightforward relationship to science capabilities than the central intensity type metrics.

Image size traditionally characterized by the $80 \%$ encircled energy diameter $\Theta(80)$ or the Full Width Half Maximum (FWHM) of the PSF. The $\Theta(80)$ metric in particular is widely used for telescope error budgeting, albeit the validity of combining $\Theta(80)$ for various error sources through RSS was rigorously proven neither analytically nor numerically.

$$
\Theta(80)_{\text {total }}^{2} \approx \sum_{i} \Theta(80)_{i}^{2}
$$

We found that the combination of $\Theta(80)^{2}$ for atmospheric seeing and various telescope errors is actually non-linear. The incremental effects of telescope aberrations highly depend on the strength of the underlying large atmospheric wavefront error (see Figure 1). Curiously, the non-linearity is definitely counter-intuitive: the $\Theta(80)$ contribution of a given telescope error is larger, if the underlying atmospheric error is worse.

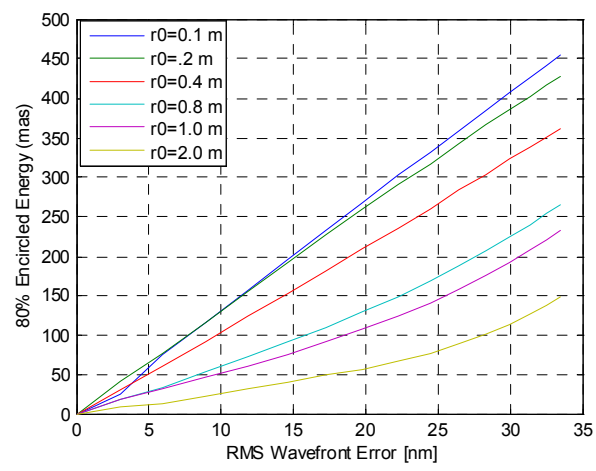

Figure 1 The $\Theta(80)$ contribution of TMT segment print through as the function of RMS wavefront error and atmospheric Fried parameter $\left(r_{0}\right)$

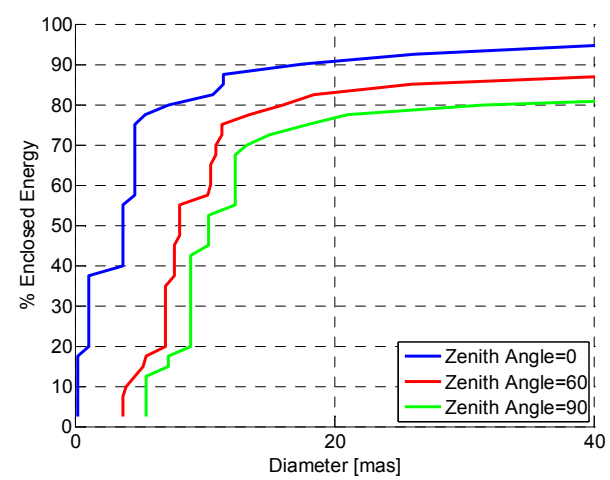

Figure 2 The encircled energy curves for TMT segment print through as the function of telescope zenith angle. 
Furthermore, for small, high spatial frequency telescope errors still showing the residuals of Airy rings, - like mirror support print through, for example, - the slope of the corresponding encircled energy curve can be very low close to the first Airy dark ring (Figure 2). This in turn leads to significant uncertainty in numerically determining $\Theta(80)$ for these errors. Of course, the effect is not physical in the sense that these small errors never occur by themselves, but nevertheless it constitutes a notable difficulty for error budgeting.

\section{THE POINT SOURCE SENSITIVITY (PSS) METRIC}

\subsection{Fourier optics interpretation of PSS}

The Fourier optics interpretation of optical systems enables the application of linear system theory concepts in optics. One of those concepts is the $\mathrm{H}_{2}$ norm [3]. It characterizes the output signal strength of a system driven with a uniform spectral intensity source. It is common to design linear controllers for actually optimizing - i.e. maximizing, - the $\mathrm{H}_{2}$ norm of the system. The class of controllers using this norm as optimization criterion is called LQG (linear quadratic Gaussian) controllers.

For a single-input-single-output system, like a telescope, it is straightforward to calculate the $\mathrm{H}_{2}$ norm $\left(\|H\|_{2}\right)$ from the transfer function of the system, in this case the $O T F$.

$$
\|\mathcal{H}\|_{2}^{2}=\int_{\infty} \mathcal{H} \mathcal{H}^{*} d v=\int_{\infty}|\mathcal{H}|^{2} d v
$$

An unresolved point target on the sky is a good realization of a uniform spatial spectral intensity source. It is reasonable then to conclude that the square of the $\mathrm{H}_{2}$ norm for the telescope is actually its Point Source Sensitivity (PSS).

\subsection{Physical interpretation of PSS}

Assuming a star irradiance (photon flux per unit collecting area) of $N_{\text {star }}$, the total signal flux collected by the telescope is

$$
\Phi_{\text {star }}=\frac{\pi}{4} D^{2} N_{\text {star }}
$$

where $D$ is the diameter of the telescope primary mirror. This signal is distributed on the focal surface according to the Point Spread Function $(P S F)$ of the optical system, $h$, which is normalized over the total sky area: $\int_{\infty} h d \Omega=1$.

On the other hand, the background sky radiant exitance (photon flux per unit sky area per unit collecting area), $N_{\text {sky }}$ can be considered spatially uniform in the vicinity of the star. The background flux contribution to the measurement depends on the sky area that needs to be included in the image processing to capture the entire signal, i.e. the Equivalent Noise Area, ENA.

$$
\Phi_{s k y}=(E N A) \frac{\pi}{4} D^{2} N_{s k y}
$$

Both the star and background fluxes follow the Poisson distribution, with identical means and variances. The measurement uncertainty, i.e. noise also depends on the integration time, $T$. It is also assumed that these two fluxes are statistically independent.

$$
\begin{aligned}
\sigma_{s t a r}^{2} & =\Phi_{s t a r} T \\
\sigma_{s k y}^{2} & =\Phi_{s k y} T \\
\sigma_{\text {meas }}^{2} & =\sigma_{s t a r}^{2}+\sigma_{s k y}^{2}
\end{aligned}
$$


By neglecting effects that can be - at least theoretically, - minimized by technology, like dark current, readout noise, and quantization noise, an upper bound for the $S N R$ can be derived [4]. In our estimate, we also ignored the uncertainty of background estimate; for reference, it is included in the SNR calculations of Mighell [5].

$$
S N R=\frac{\Phi_{\text {star }} T}{\sigma_{\text {meas }}}=\frac{\Phi_{\text {star }}}{\sqrt{\Phi_{\text {star }}+\Phi_{\text {sky }}}} \sqrt{T}
$$

King [6] and later others, like Mighell [5] showed that the appropriate area to be considered depends on the shape of the PSF only.

$$
E N A=\frac{1}{\int_{\infty}|h|^{2} d \Omega}
$$

Since the $O T F$ and PSF are Fourier transform pairs, the ENA is also the inverse of the PSS, as it is defined in Equation(2.1).

$$
\begin{gathered}
\int_{\infty}|\mathcal{H}|^{2} d v=\int_{\infty}|h|^{2} d \Omega \\
E N A=\frac{1}{P S S}
\end{gathered}
$$

Using Equations (2.3), (2.5), and (2.7), a lower bound for the integration time required to achieve a given signal to noise ratio can be determined.

$$
T=\frac{S N R^{2}}{\Phi_{\text {star }}^{2}}\left[\Phi_{s t a r}+\Phi_{s k y}\right]=\frac{S N R^{2}}{\left(\frac{\pi}{4} D^{2}\right) N_{s t a r}^{2}}\left[N_{s t a r}+\frac{N_{s k y}}{P S S}\right]
$$

For observations of faint stars, where the background is the dominant error, the integration time is clearly proportional to the ENA.

$$
\begin{aligned}
& T \approx \frac{S N R^{2} N_{s k y}}{\left(\frac{\pi}{4} D^{2}\right) N_{s t a r}^{2}} \frac{1}{P S S} \propto E N A, \text { assuming } \\
& N_{s k y} \gg(P S S) N_{s t a r}
\end{aligned}
$$

If we want to characterize and evaluate an optical imaging system, like a telescope with the lower bound of the integration time it enables, it is useful to establish a baseline the various potential realizations of the system can be compared to. By picking the perfect, unperturbed telescope at its particular site as baseline, we can use the corresponding $P S S_{\text {atmosphere }}$, - which is clearly the highest possible PSS for the given system, - to normalize the metric. For a ground based astronomical telescope, $P S S_{\text {atmosphere }}$ includes atmospheric seeing, inherent diffraction effects, and potential design aberrations, but not the implementation errors. $P S S_{\text {actual }}$ characterizes the combined system of imperfect telescope and atmosphere.

$$
T_{\text {actual }}=\frac{T_{\text {perfect }}}{P S S N} \text { where } P S S N=\frac{P S S_{\text {actual }}}{P S S_{\text {atmosphere }}} \leq 1
$$

Equation (2.10) defines the Normalized Point Source Sensitivity (PSSN), which is used for (i) characterizing the performance of the Thirty Meter Telescope and (ii) allocating its performance to the various subsystems through an error budget.

It is worth to mention that - as it is shown in Equation (2.8), - the integration time is inversely proportional to $D^{2}$, which is one of the major driving forces behind the quest for larger and larger telescopes. 


\section{RELATIONSHIP TO OTHER METRICS}

\subsection{Strehl Ratio}

The long exposure (time averaged) OTF is the autocorrelation of the pupil function [7]. It can be shown that for normally distributed, zero mean, homogenous, and isotropic wavefront errors, the long exposure OTF can be approximated as pupil diffraction and aberration terms [8]. The aberration is characterized with the phase structure function $\mathscr{D}$ of the pupil.

$$
\mathcal{H} \approx e^{-\frac{1}{2} \mathscr{D}} \mathcal{H}_{\text {diffraction }}
$$

Furthermore, as the (i) atmospheric wavefront error can be approximated as homogenous and isotropic, (ii) the structure function for statistically independent aberrations is additive (the $O T F$ is multiplicative), and (iii) the phase structure function is by definition bounded by the phase variance $\left(\mathscr{D}<2 \sigma_{\phi}^{2}\right)$, the Point Source Sensitivity of the system is bounded by the product of the joint $\mathrm{H}_{2}$ norm of the perfect (diffraction limited) telescope with atmospheric seeing, and the Strehl Ratio of the telescope itself.

$$
\begin{gathered}
\|\mathcal{H}\|_{2}^{2} \approx \int_{\infty} e^{-\left(\Phi_{a t m}+\Phi_{l e l}\right)}\left|\mathcal{H}_{\text {diff }}\right|^{2} d v>e^{-2 \sigma_{\phi}^{2}} \int_{\infty} e^{-\Phi_{a t m}}\left|\mathcal{H}_{\text {diff }}\right|^{2} d v \\
P S S>S^{2}\left\|\mathcal{H}_{a t m}\right\|_{2}^{2}
\end{gathered}
$$

In turn it means the Normalized Point Source Sensitivity is bounded by the Strehl Ratio of the telescope.

$$
P S S N>S^{2} \approx e^{-2 \sigma_{\phi}^{2}} \approx 1-2 \sigma_{\phi}^{2}
$$

Both Equations (3.2) and (3.3) assume telescope aberrations small enough to permit the Maréchal approximation. However, in general the telescope aberrations are not just not small enough; they are also not necessarily normally distributed, homogenous and isotropic, either. Nevertheless, it can be show [9] that Equation (3.3) is true in general.

\subsection{RMS wavefront error}

Extensive analytical and numerical studies [9] support an extension to the approximation in Equation (3.3).

$$
\begin{aligned}
& P S S N \approx 1-\alpha \sigma_{\phi}^{2} \\
& \alpha<2
\end{aligned}
$$

As it is shown in Section 4, the $\alpha$ coefficient defined here with dimension of $1 / \mathrm{rad}^{2}$ is the function of wavelength $(\lambda)$, atmospheric seeing $\left(r_{0}\right)$, and the spatial frequency of the wavefront error. However, for a sufficient range it is independent of the error strength.

The approximation in Equation (3.4) enables the estimation of PSSN by Zernike components of the OPD. For a given atmospheric seeing, a unique $\alpha_{i}$ coefficient is assigned to each Zernike term with RMS wavefront error of $\sigma_{\phi i}$. As it is shown in [9], as well as in Section 4.1, the PSSN values are multiplicative for independent errors.

$$
P S S N \approx \prod_{i}\left(1-\alpha_{i} \sigma_{\phi i}^{2}\right)
$$

The approximation is also valid for any other independent basis, like the Zernike expansion of systematic primary mirror segment figure errors [10]. It is also very useful for estimating the performance of (optical) modal controllers in the active optics system.

\section{$3.380 \%$ encircled energy diameter}

For the general case the relationship between $\Theta(80)$ and $E N A$ is not steady; it depends considerably on the actual shape of the PSF. While the conversion is straightforward (see Equation (3.7)), it has to be evaluated on a case by case basis. For example, it varies from atmospheric seeing to Gaussian $P S F$ to a simple focus error [11]. 


$$
\begin{aligned}
E N A_{\text {atmosphere }} & =0.807 * \Theta(80)_{\text {atmosphere }}^{2} \\
E N A_{\text {Gaussian }} & =0.956 * \Theta(80)_{\text {Gaussian }}^{2} \\
E N A_{\text {focus }(2,0)} & =1.02 * \Theta(80)_{\text {focus }(2,0)}^{2}
\end{aligned}
$$

These variations demonstrate that - unlike the $E N A,-\Theta(80)$ is not a metric directly related to science capabilities. While it is suitable for "ballpark" estimates, the associated error can be substantial.

Furthermore, for an arbitrary $P S F$, summing $\Theta(80)^{2}$ or $E N A$ of various aberrations also carries noticeable approximation errors (see Section 4.1). This in turn makes a term-by-term approach, like Equation (3.5) for PSSN, error prone and not practical for engineering calculations.

It is worth to mention that some special cases behave more regularly, though. Such an example is the Gaussian PSF with $R M S$ width of $\delta[12]$.

$$
\begin{aligned}
& h_{\text {Gaussian }}(\rho)=\frac{1}{2 \pi \delta^{2}} e^{-\frac{\rho^{2}}{2 \delta^{2}}} \\
& \Theta(80)_{\text {Gaussian }}=(2 \sqrt{2 \ln 5}) \delta \\
& \text { ENA }_{\text {Gaussian }}=4 \pi \delta^{2}=\frac{\pi}{2 \ln 5} \Theta(80)_{\text {Gaussian }}^{2}
\end{aligned}
$$

Since the convolution of two Gaussian PSFs is also a Gaussian PSF with $\delta^{2}=\delta_{1}^{2}+\delta_{2}^{2}$, for this special case the Equivalent Noise Areas are happen to be additive.

\section{KEY FEATURES OF THE PSSN METRIC}

\subsection{Multiplicative error budgeting}

One of the key features making PSSN so useful for systems engineering is its multiplicative property. Thorough analytical and numerical investigation of this property was provided by Seo et al.[9], proving the exceptional capability of PSSN for multiplicative error budgeting. Here we show an illustrative example only.

Table 1 shows the estimation errors associated with multiplicatively combining PSSN, compared to the estimation error of additively combining ENA for the same set of telescope implementation errors [13]: (i) telescope zenith angle $(z a)$ dependent segment support print through, and (ii) segment shape residual error after polishing. Both of these errors are small compared to the also included atmospheric seeing $\left(r_{0}\right)$.

Table 1 Relative errors associated with combining PSSN or ENA for the same aberrations

\begin{tabular}{|c|c|c|c|c|c|c|}
\hline \multirow{2}{*}{ PSSN } & ENA & \multicolumn{2}{|c|}{$z a=10^{\circ}$} & \multicolumn{2}{c|}{$z a=30^{\circ}$} & \multicolumn{2}{c|}{$z a=50^{\circ}$} \\
\hline$r_{0}=10 \mathrm{~cm}$ & $0.28 \%$ & $3.0 \%$ & $0.32 \%$ & $3.7 \%$ & 0.325 & $4.7 \%$ \\
\hline$r_{0}=15 \mathrm{~cm}$ & $0.48 \%$ & $4.1 \%$ & $0.50 \%$ & $5.2 \%$ & $0.57 \%$ & $6.8 \%$ \\
\hline$r_{0}=20 \mathrm{~cm}$ & $0.66 \%$ & $5.2 \%$ & $0.70 \%$ & $6.8 \%$ & $0.72 \%$ & $8.8 \%$ \\
\hline$r_{0}=25 \mathrm{~cm}$ & $0.87 \%$ & $6.3 \%$ & $0.90 \%$ & $8.1 \%$ & $0.91 \%$ & $10.6 \%$ \\
\hline
\end{tabular}

The uncertainty associated with combining $P S S N$ values is consistently smaller by about an order of magnitude than the uncertainty for combining ENA. This demonstrates that, while summation of ENA may be suitable for "ballpark" estimates, in rigorous error budgeting the large number of effects to be combined in ENA domain can drive the overall uncertainty of such combination unacceptably high. 


\subsection{Spatial frequency and atmospheric seeing dependence}

In order to demonstrate the effects of the (i) spatial frequency of the aberration, as well as the (ii) strength of atmospheric seeing on the $\alpha$ coefficient, random, homogenous, and isotropic telescope aberrations were considered with RMS phase error of $\sigma_{\phi}$ and correlation length of $\sigma_{d}$ [9]. The atmospheric seeing it was combined with is characterized by its $r_{0}$.

$$
\mathcal{D}_{\text {telescope }}(r)=\sigma_{\phi}^{2}\left(1-e^{-\frac{r^{2}}{\sigma_{d}^{2}}}\right)
$$

Important features of PSSN and the $\alpha$ coefficient can be deduced from Figure 3:

i. As expected, the optical effects of higher spatial frequency (shorter correlation length) phase errors are more prominent, i.e. the corresponding $\alpha$ coefficient is higher;

ii. The $\alpha$ coefficient is independent of the strength of the phase error for practical telescope aberrations, i.e. when the dominant effect is atmospheric seeing $\left(r_{0}<<\sigma_{d}\right)$;

iii. Following our intuition, as atmospheric seeing diminishes ( $r_{0}$ increases), a given telescope aberration affects PSSN more severely (through increased $\alpha$ coefficient). In other words, telescope errors are more noticeable, when the atmospheric seeing is excellent;

iv. The $\alpha$ coefficient is saturating to 2, as expected from Equation (3.4), for large $r_{0}$ (weak atmospheric seeing) and small, high spatial frequency telescope aberrations $\left(r_{0} \gg \sigma_{d}\right)$.
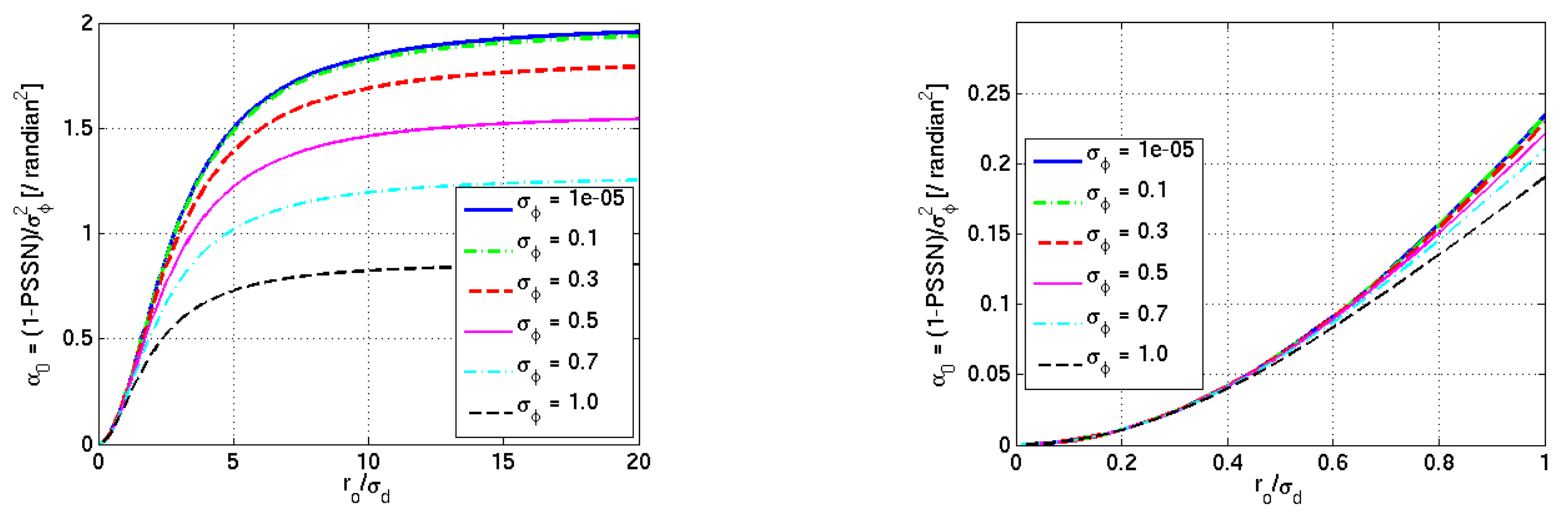

Figure 3 Spatial frequency dependence of the $\alpha$ coefficient ( $\sigma_{\phi}^{2}$ is the wavefront variance in radian, $\sigma_{d}$ is the correlation length of the wavefront error, and $r_{0}$ is the atmospheric Fried parameter). The graph on the right is the zoomed-in version of the graph on the left.

\subsection{Wavelength dependence}

The PSSN metric, just like any other image quality metric, profoundly depends on the wavelength of light it is evaluated for. This characteristic is properly reflected in the wavelength dependence of the $\alpha$ coefficient [14]. As Figure 4 indicates, the coefficient increases with wavelength in different ways for different aberrations, but without exception it does increase. It also saturates at or close to 2 , as expected.

While $\alpha$ saturates for longer wavelength, the phase error is clearly diminishes, which in turn leads to the intuitive conclusion: for longer wavelengths, PSSN saturates to 1. 


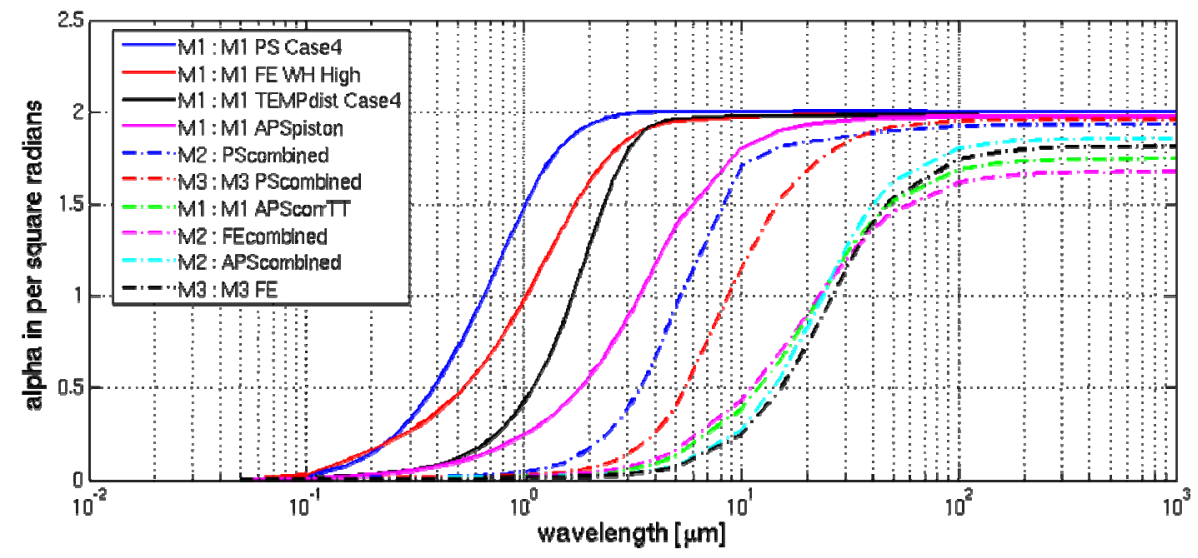

Figure 4 Wavelength dependence of the $\alpha$ coefficient

\section{EXTENSIONS TO THE PSSN METRIC}

\subsection{Field dependence}

The expected image quality of the optical designs for ground based astronomical telescopes - just like that of many other optical systems - usually depends on the image position in the Field of View (FOV). These design aberrations can occasionally be significant compared to the optical consequences of manufacturing and alignment imperfections captured in the error budget. While the science impact of the design aberrations in itself can be an important consideration, it may mask other substantial effects. Consequently, the off-axis extension to the PSSN definition must distinguish between design aberrations and realization imperfections.

Seo et al. [15] introduced such an extension: design indicates design (inherent) field dependent aberrations combined with the atmospheric seeing, while $\varphi$ denotes the field point PSS is calculated for.

$$
P S S N=\frac{P S S_{\text {actual }, \varphi}}{P S S_{\text {artmosphere }, \varphi=0}}=\frac{P S S_{\text {actual }, \varphi}}{P S S_{\text {design }, \varphi}} \frac{P S S_{\text {design }, \varphi}}{P S S_{\text {atmospher }, \varphi=0}}=P S S N_{F} * P S S N_{N}
$$

$P S S N_{N}$ is normalized at any field point to the on-axis unperturbed telescope performance, while $P S S N_{F}$ uses as reference the unperturbed telescope performance at the same field point. The different behavior of these metrics is demonstrated by Figure 5.
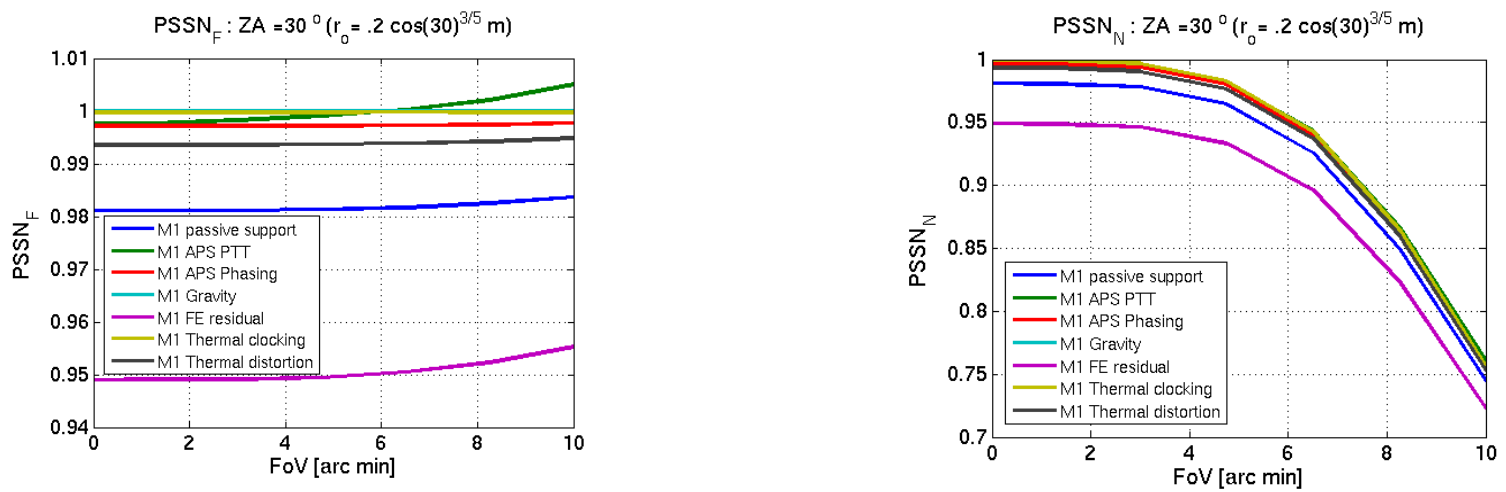

Figure 5 Comparison of the two components of the off-axis PSSN for various TMT primary mirror (M1) errors (telescope zenith angle is $30^{\circ}, r_{0}$ in zenith direction is $20 \mathrm{~cm}$ ) 
As Figure 5 indicates, $\operatorname{PSSN}_{F}$ is reasonably constant throughout the field of view of the telescope. Some errors are slightly improving with field angle due to mode cancellation, i.e. the astigmatism content of those errors is cancelled out by the field dependent design astigmatism. However, by picking $\operatorname{PSSN}_{F}$ as performance metric, the error budget becomes practically independent of field angle. Furthermore, it can be shown (Figure 6) that $\operatorname{PSSN}_{F}$ is a multiplicative metric with very good approximation $(<0.3 \%)$, just as $P S S N$ is.

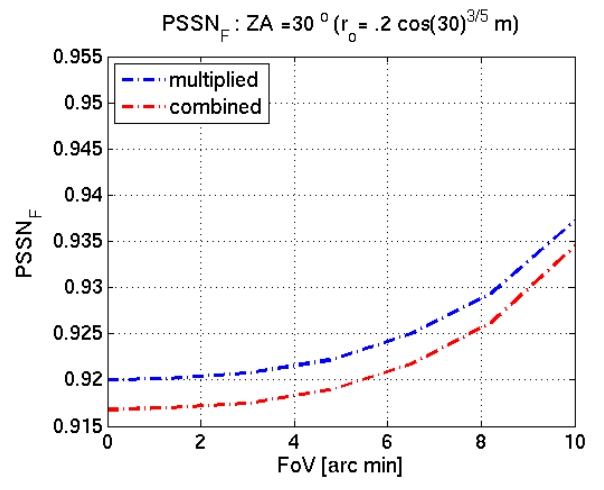

Figure 6 Combined and multiplied $P S S N_{F}$ values for the same set of TMT primary mirror (M1) errors as in Figure 5

\subsection{Throughput and vignetting}

In Section 2.2, for the sake of simplicity we neglected the contribution of telescope throughput, $\mathcal{T}$ to the integration time. By including it in Equation (2.5), it becomes evident that the integration time is inversely proportional to the throughput.

$$
T=\frac{S N R^{2}}{\mathcal{T} \Phi_{\text {star }}^{2}}\left[\Phi_{\text {star }}+\Phi_{\text {sky }}\right]
$$

At the limit of dominant background, the effect of throughput is analogous to that of PSS, which in turn enables a normalization similar to $P S S N$, i.e. normalization to the throughput of the unperturbed telescope, $\tau_{\text {perfect }}$.

$$
\begin{aligned}
& T \propto \frac{1}{\mathcal{T}(P S S)} \\
& T_{\text {actual }}=\frac{T_{\text {perfect }}}{\mathcal{T}_{N} P S S N}, \text { where } \tau_{N}=\frac{\mathcal{T}_{\text {actual }}}{\mathcal{T}_{\text {perfect }}}
\end{aligned}
$$

This uniform handling of image quality $(P S S N)$ and normalized throughput $\left(\mathcal{T}_{N}\right)$ provides a tool to compare various telescope errors in the same framework. For example, in case of a segmented primary mirror, a defective segment actuator usually mitigated by temporarily "tilting out" the affected segment, in effect removing it from light collection. Such a missing segment causes diffraction effects, but the major error contribution is reduced throughput [16]. The evaluation of the science impact is straightforward in the framework described.

A more common example can be vignetting. It again has both diffraction and throughput consequences that can individually be compared to other effects degrading science sensitivity of the telescope. Figure 7 shows the field dependent vignetting of the Thirty Meter Telescope in comparison to its field dependent astigmatism inherent to the Ritchey-Crétien design. The figure visually demonstrates the reasoning behind the design decision allocating 15 arcmin unvignetted FOV, albeit the scientifically useful, unobstructed FOV of the telescope is 20 arcmin: for large field angles, the effect of slight vignetting is negligible compared to the science consequence of design astigmatism.

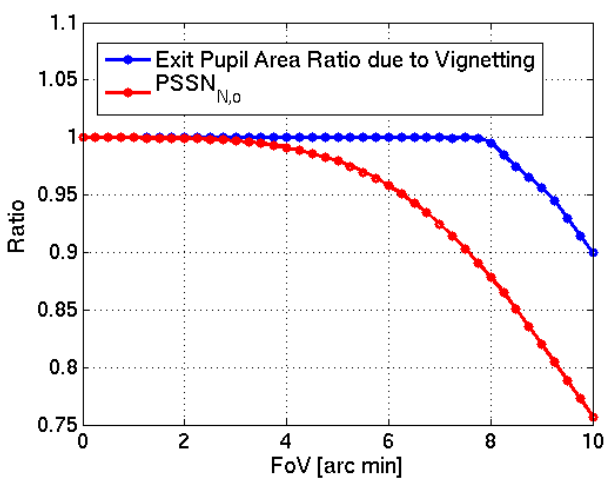

Figure 7 Comparison of the science impact of field dependent vignetting and design astigmatism of the Thirty Meter Telescope [15] 


\section{CONCLUSION}

The Thirty Meter Telescope Observatory uses the normalized Point Source Sensitivity as its major image quality metric for atmospheric seeing limited observations. The metric takes advantage of the Equivalent Noise Area concept, as well as the atmospheric normalization featured by the Central Intensity Ratio.

The PSSN metric is directly related to the science integration time increase due to telescope errors in background limited observations of point targets. As it is an image size type metric, it is also a good predictor of spectroscopic efficiency [17].

The metric is multiplicative for various statistically independent errors with good approximation, and as such it can be used for engineering error budgeting. Regarding error budgets, it is worth to mention that a PSSN budget automatically allows increasing telescope aberrations with increasing zenith angles, following the atmospheric seeing degradation away from zenith direction.

Although the direct calculation of PSSN is somewhat involved, as it requires the evaluation of the OTF as well as the $P S F$ of the telescope, there are good approximation methods for estimating it. The metric accounts for the strength and spatial frequency content of the aberration; it depends on the actual atmospheric seeing and the wavelength of the light.

The PSSN metric can be extended for off-axis imaging in a straightforward manner. In turn, the off-axis metric enables the extension of multiplicative error budgeting throughout the entire field of view, regardless of possible field dependent design aberrations. Similarly, it was shown that throughput degradation, like vignetting, surface or reflection losses can be interpreted in the same framework and their science efficiency impact can be directly compared to that of image quality $(P S S N)$ degradation.

\section{ACKNOWLEDGMENTS}

The TMT Project gratefully acknowledges the support of the TMT partner institutions. They are the Association of Canadian Universities for Research in Astronomy (ACURA), the California Institute of Technology and the University of California. This work was supported as well by the Gordon and Betty Moore Foundation, the Canada Foundation for Innovation, the Ontario Ministry of Research and Innovation, the National Research Council of Canada, the Natural Sciences and Engineering Research Council of Canada, the British Columbia Knowledge Development Fund, the Association of Universities for Research in Astronomy (AURA) and the U.S. National Science Foundation.

\section{REFERENCES}

[1] P. Dierickx, "Optical Performance of Large Ground-based Telescopes," Journal of Modern Optics, 39(3), 569588 (1992).

[2] J. Castro, C. D. Bello, L. Jochum et al., "Image quality and active optics for the Gran Telescopio Canarias," Proceedings of the SPIE, 3352, 386 (1998).

[3] W. S. Levine, [The Control Handbook] CRC Press, Boca Raton(1995).

[4] S. B. Howell, [Handbook of CCD astronomy] Cambridge University Press, Cambridge, 54 (2000).

[5] K. J. Mighell, "Stellar photometry and astrometry with discrete point spread functions," Mon. Not. R. Astron. Soc., 361, 861-878 (2005).

[6] I. R. King, "Accuracy of measurement of star images on a pixel array," Publications of the Astronomical Society of the Pacific, 95(February), 163-168 (1983).

[7] J. W. Goodman, [Introduction to Fourier Optics] McGrow-Hill, New York(1996). 
[8] B. Ellerbroek, "OTF and Structure Function Methods for (Seeing-Limited) TMT Error Budgets," TMT Report, TMT.SEN.PRE.05.002, (2005).

[9] B.-J. Seo, C. Nissly, G. Z. Angeli et al., "Analysis of normalized point source sensitivity as a performance metric for large telescopes," Applied Optics, 48(31), 5997-6007 (2009).

[10] B.-J. Seo, C. Nissly, M. Troy et al., "Segmented Zernike study on the Thirty Meter Telescope," TMT Report, TMT.SEN.TEC.08.048, (2008).

[11] T. Mast, "Concerns about the TMT seeing-limited error budget," personal communication, (2008).

[12] G. Z. Angeli, “Seeing versus Exit Pupil OPD,” TMT Report, TMT.SEN.TEC.05.001, (2005).

[13] G. Z. Angeli, “Performance Metric Test,” TMT Report, TMT.SEN.TEC.08.010, (2008).

[14] B.-J. Seo, C. Nissly, M. Troy et al., "PSSN dependency on wavelength for the Thirty Meter Telescope," TMT Report, TMT.SEN.TEC.08.047, (2008).

[15] B.-J. Seo, C. Nissly, M. Troy et al., "Normalized point source sensitivity for off-axis optical performance evaluation of the Thirty Meter Telescope," Proceedings of the SPIE, 7738, (2010).

[16] C. Nissly, B.-J. Seo, M. Troy et al., "TMT segment removal PSSN impact study," TMT Report, TMT.SEN.TEC.09.013, (2009).

[17] M. Schöck, “TMT spectroscopy performance investigation,” TMT Report, TMT.SEN.TEC.09.016, (2009). 